\title{
Hemodynamic effects of the carotid abnormalities courses by MRI and ultrasound
}

\author{
Yuliya Stankevich ${ }^{1 *}$, Mariya Rezakova², Bogomyakova Olga', Liubov Shraibman¹, Andrey Tulupov ${ }^{1,3}$ \\ From 18th Annual SCMR Scientific Sessions \\ Nice, France. 4-7 February 2015
}

\section{Background}

The aim of our investigation was to determine effect of the internal carotid arteries (ICAs) tortuosity (S-shape), kinking and coiling on the arterial blood flow by magnetic resonance imaging and ultrasound (US).

\section{Methods}

50 healthy volunteers (control group) and 43 patients with carotid abnormalities (CAs) were examined on 1.5T MR-scanner using routine MR protocol and quantitative MR angiography (qMRA) for estimate of the arterial blood flow velocity and cross sectional area of the ICA. We observed the cross sectional area and the values of peak velocity, mean velocity, flux velocity of arterial blood flow at one cardiac cycle at cervical and intracranial segments of the ICA (Fig. 1). 20 volunteers were performed US for the assessment of the vascular wall and peak systolic velocity (PSV) values of the arterial blood at the portion between common carotid artery (CCA) bifurcation and ICA. Statistical analysis was performed by mean value and confidence interval (for $\mathrm{p}=0.05$ ). Student's paired $\mathrm{t}$-test was used to indicate significant differences between mean values at the patient and control groups.

\section{Results}

The shapes of the graphs of the mean velocity depending on the phase of the cardiac cycle were the same in

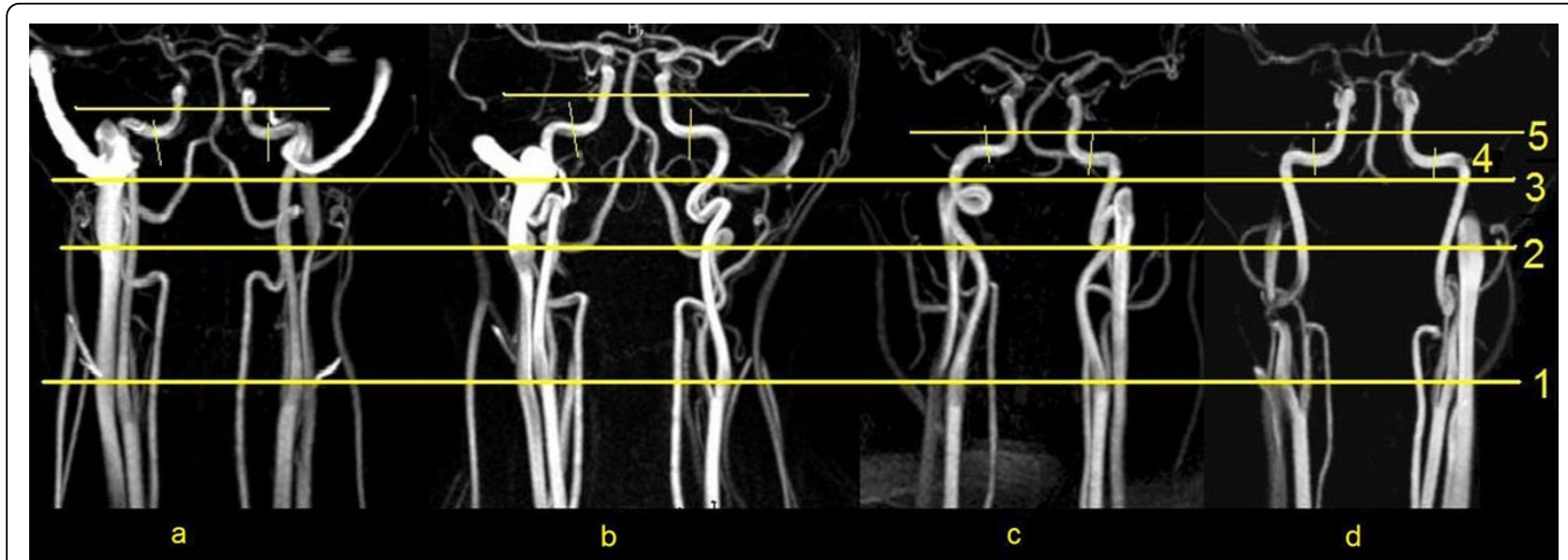

Figure 1 Investigated ICA segments in normal course (a) and abnormalities course (b - S-shape, c - coiling, d - kinking): 1 - C1, cervical segment (proximal part); 2 - C1, cervical segment (middle part); 3 - C2, petrous segment (vertical part); 4 - C2, petrous segment (horizontal part); 5 - C5, cavernous segment.

IInternational Tomography Center SB RAS, Novosibirsk, Russian Federation Full list of author information is available at the end of the article 


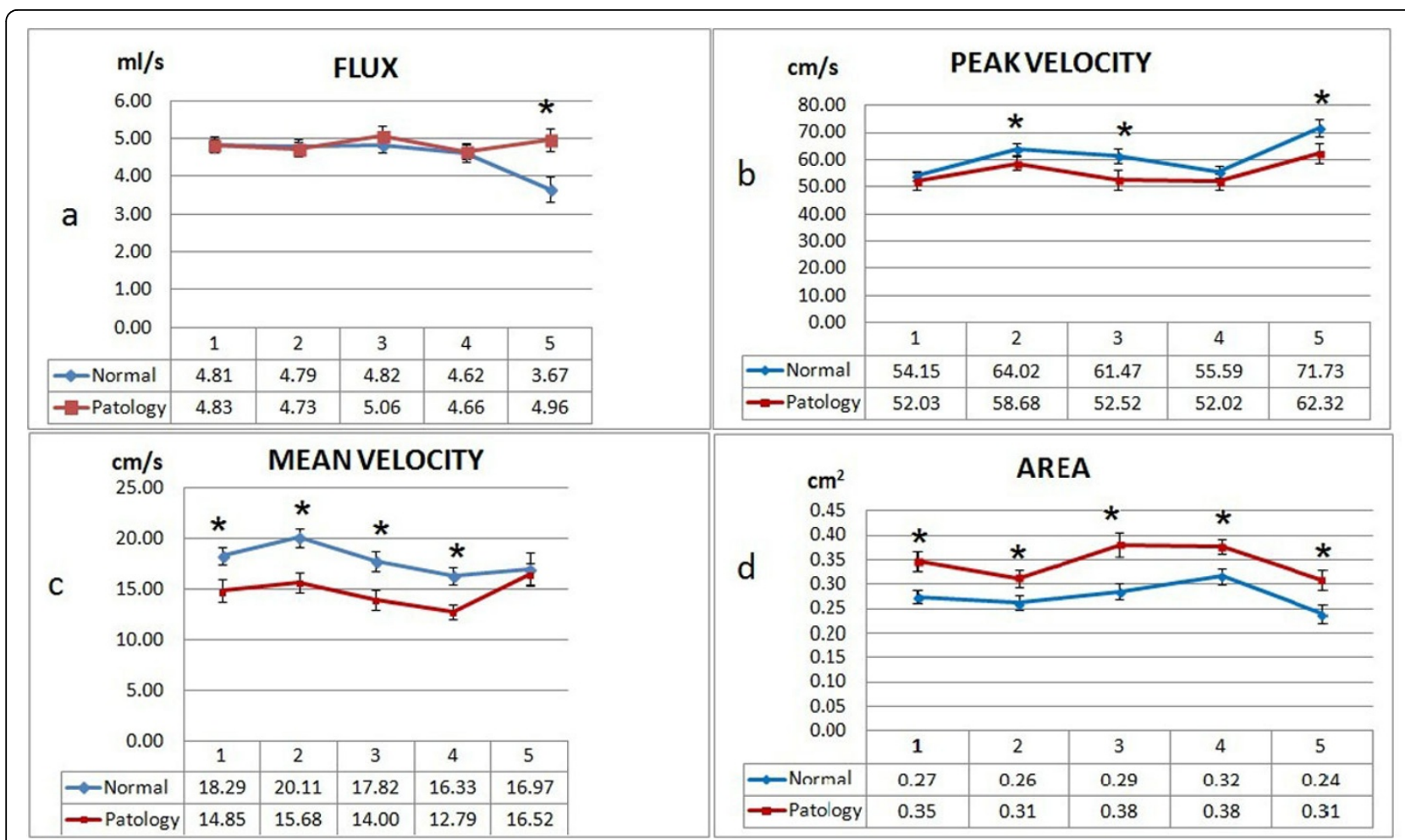

Figure 2 Cross-sectional area (d) and arterial blood flow velocity parameters (a - flux velocity, b - peak velocity, c - mean velocity) at different ICA segments. * - significant differences between values cross-sectional area and arterial blood flow velocity parameters of the ICA at control groups $(n=100)$ and CAs group $(n=67), p=0,05$

the case of the control and CAs groups obtained by MRI and US. The values of blood flow velocity parameters were significant differences at different segments of the ICA in the control and CAs groups (Fig. 2). There were no significant differences between values of the arterial blood velocity parameters in case of the tortuosity (S-shape), kinking and coiling at different segments of ICA. The cross sectional area of the vessel was significantly higher at the all investigated parts in case of CAs (Fig. 2). The correlation coefficient between the values of peak systolic velocity obtained by ultrasound and MRI was low (-0.01).

\section{Conclusions}

Type of the artery pulsation at CAs is the same as the control group and corresponds to the musculo-elastic arteries. CAs affects the hemodynamic throughout the artery whith a background of increasing the area of its cross section. The ultrasound has confirmed the results obtained on MRI qualitatively, but correlation coefficient for quantitative parameters was low.

\section{Funding}

This work was financially supported by the Russian Science Foundation (project \# 14-35-00020).

\section{Authors' details}

'International Tomography Center SB RAS, Novosibirsk, Russian Federation. ${ }^{2}$ Institute of Physiology and Fundamental Medicine SB RAMS, Novosibirsk, Russian Federation. ${ }^{3}$ Novosibirsk State University, Novosibirsk, Russian Federation.

Published: 3 February 2015

doi:10.1186/1532-429X-17-S1-P415

Cite this article as: Stankevich et al:: Hemodynamic effects of the carotid abnormalities courses by MRI and ultrasound. Journal of Cardiovascular Magnetic Resonance 2015 17(Suppl 1):P415.

\section{Submit your next manuscript to BioMed Central} and take full advantage of:

- Convenient online submission

- Thorough peer review

- No space constraints or color figure charges

- Immediate publication on acceptance

- Inclusion in PubMed, CAS, Scopus and Google Scholar

- Research which is freely available for redistribution 